\title{
Aerosol delivery via noninvasive ventilation: role of models and bioanalysis
}

\author{
Haitham Saeed, Hadeer S. Harb, Yasmin M. Madney, Mohamed E. A. Abdelrahim \\ Clinical Pharmacy Department, Faculty of Pharmacy, Beni-Suef University, Beni-Suef, Egypt \\ Contributions: (I) Conception and design: MEA Abdelrahim; (II) Administrative support: All authors; (III) Provision of study materials or patients: All \\ authors. (IV) Collection and assembly of data: HS Harb, H Saeed, YM Madney; (V) Data analysis and interpretation: All authors; (VI) Manuscript \\ writing: All authors; (VII) Final approval of manuscript: All authors. \\ Correspondence to: Mohamed E. A. Abdelrahim. Professor, Department of Clinical Pharmacy, Faculty of Pharmacy, Beni-suef University, Beni-suef, \\ Egypt. Email: mohamedemam9@yahoo.com.
}

\begin{abstract}
Non-invasive ventilation (NIV) is external support for respiration to assist breathing in case of respiratory failure (either hypercapnic or hypoxemic) without patient intubation. Nowadays, medicated aerosols are normally delivered to mechanically ventilated patients by nebulizers and pressurized metereddose inhaler (pMDI) attached to adapter or spacer that fit into the ventilated circuit. Studies with obstructive lung disease patients have shown that aerosol delivery during mechanical ventilation is possible and of benefit. There are several models for investigating the aerosol delivery and deposition during mechanical ventilation such as in vitro, in vivo, and ex vivo models, these models depend on the technique used for quantitative or qualitative measurement of the deposited aerosol. In vitro models could be used for calculating the total emitted doses from different aerosol-generating devices or for aerodynamic characterization of the deposited inhaled medications. In vivo models dependents of extracting drugs from biological samples for measuring its concentration and bioavailability (pharmacokinetic model) or be dependent on the imaging technique of the radioactive aerosol. Applying different methods to predict aerosol efficiency before starting NIV and to quantify aerosol delivery during NIV are promising approaches that guide clinicians to avoid treatment failure before and during patient therapy.
\end{abstract}

Keywords: Aerosol therapy; non-invasive ventilation; interfaces; nebulizer; pressurized metered-dose inhaler (pMDI)

Submitted Feb 03, 2020. Accepted for publication Sep 11, 2020.

doi: $10.21037 /$ atm-20-1261

View this article at: http://dx.doi.org/10.21037/atm-20-1261

\section{Introduction}

Pulmonary diseases are selectively treated by inhaled drugs which are considered a superior route of drug administration over parenteral routes due to rapid drug effect, higher local pulmonary drug delivery with minimal systemic adverse actions, and requiring lower drug doses $(1,2)$. Non-invasive ventilation (NIV) is external support applying positive pressure ventilation to assist breathing in case of respiratory failure (either hypercapnic or hypoxemic) without a need for patient intubation (3). Interruption of NIV to administer aerosol therapy is associated with adverse effects for the patient. Introducing inhaled medication during mechanical ventilation can achieve effective lung levels of medication with clinical effects comparable to levels reported in spontaneously breathing patients. This is also the case when applied for critically ill patients receiving NIV. Several models with different analysis techniques are available for determination of the efficiency and efficacy of aerosol-generating devices through measuring aerosol deposition and aerodynamic characterization of the emitted drug. These models (in vitro, ex vivo, and in vivo) help determine the effect of different factors in aerosol delivery 
and guiding to optimize aerosol therapy (4-6). Many factors influence the deposition of inhaled drugs in the lungs during NIV including; type and position of an aerosol generator, gas density, and humidity of the circuit, the characteristics of aerosolized particles, type of patient interface, ventilator parameters and some factors related to the patient (7). This review aims to introduce methods of testing aerosol delivery and the role of modeling and bioanalysis in addressing these factors and how they influence the efficiency of aerosol delivery, with a review of available in vitro and in vivo studies to optimize ongoing clinical benefits.

\section{Methods used for aerosol delivery quantification during NIV}

\section{In vitro methods}

These methods simulate aspects of aerosol delivery with conditions and characteristics designed to study specific discrete on the bench that cannot be directly observed with patients. They aim at characterizing aerosol produced by various devices with the aerodynamic characterization of an aerosolized particle with an evaluation of how those particles transit and deposit through various pathways under different conditions, besides determining the amount of delivered aerosol by certain aerosol devices.

Saeed $e t a l$. tested the effect of different nebulizer designs on the aerosol delivery from each design during NIV, using a breathing simulator to mimic the patient breathing pattern (8). This in vitro study showed that different nebulizer designs of jet nebulizers (3 designs) have a significant effect on the aerosol delivery, while all tested designs (3 designs) of VMN are similar in efficacy (8). In this study, the comparison between different nebulizers was judged through the total emitted dose and dead volume (amounts of the drug remaining inside the nebulizer chamber after the end of nebulization).

\section{In vitro determination of emitted dose}

The operation of these techniques depends on the presence of three essential components; inhaler device (aerosolgenerating device), ventilator, and breathing simulator (9). There are various ventilation modes and are differentially chosen to accommodate the patient condition where two inhaler types can be used for aerosol delivery within NIV circuits; nebulizers and pressurized metered-dose inhalers (pMDIs) (10). The existence of a breathing simulator machine within this model is intended to mimic the actions of the lung when an aerosol is being inhaled where this breathing machine can exhibit different inhalation/ exhalation ratios to suit the simulated patient population. Quantification of the delivered drug fraction is the target of these models which is achieved by collecting the inhaled dose emitted on a filter that should be directly placed before the simulator to estimate the inhaled aerosol (Figure 1). The exhaled aerosol can be estimated in a single limb circuit by placing a filter above the expiration port mounted on a pump that works to withdraw air at an estimated flow rate of $25 \mathrm{~L} / \mathrm{min}$ while in dual limb circuit; its position is at the end of the exhalation limb (Figure 1).

An in vitro study by Abdelrahim et al. was designed to compare the efficacy of different nebulizers for aerosol delivery, also the effect of nebulizer position during single limb NIV (11). Nebulizers were placed in 2 different positions; before and after the expiration port in a standard single limb NIV circuit with inspiratory-expiratory pressures of 20 and $5 \mathrm{cmH}_{2} \mathrm{O}, 15$ breaths/minute, and tidal volume of $500 \mathrm{~mL}$ (11). This in vitro model has been used in several other studies, e.g., to compare delivery from pMDI, VMN and Jet nebulizer (12), effect of fill volume placed in VMN and Jet nebulizer and humidity of the ventilation circuit while delivering (13), combining pMDI to a nebulizer in the same NIV, setting (14) and delivery in high flow nasal cannula setting (15). This model was also used to examine the influence of changing interfaces on aerosol delivery within high flow oxygen setting in adults (6).

\section{In vitro particle size characterization via inertial impaction}

Among the particle size characteristics determined by these models are the mass median aerodynamic diameter (MMAD) and particle size distribution. where particles less than $5 \mu \mathrm{m}$ are thought to reach deep pulmonary regions but those of $5 \mu \mathrm{m}$ or more are preferably deposited in the oropharyngeal region, not the lungs. These in vitro models depend on the presence of a distinct device called cascade impactor to determine the particle characteristics and the aerosol particle size distribution like Next-generation impactor (NGI) and Anderson cascade impactor (ACI). Precisely, these devices can assess the particle size and define the range of aerosolized particles within the inhaled dosage form. The in vitro particle size characterization model simply contains an electrostatic filter in a holder, breathing 
A

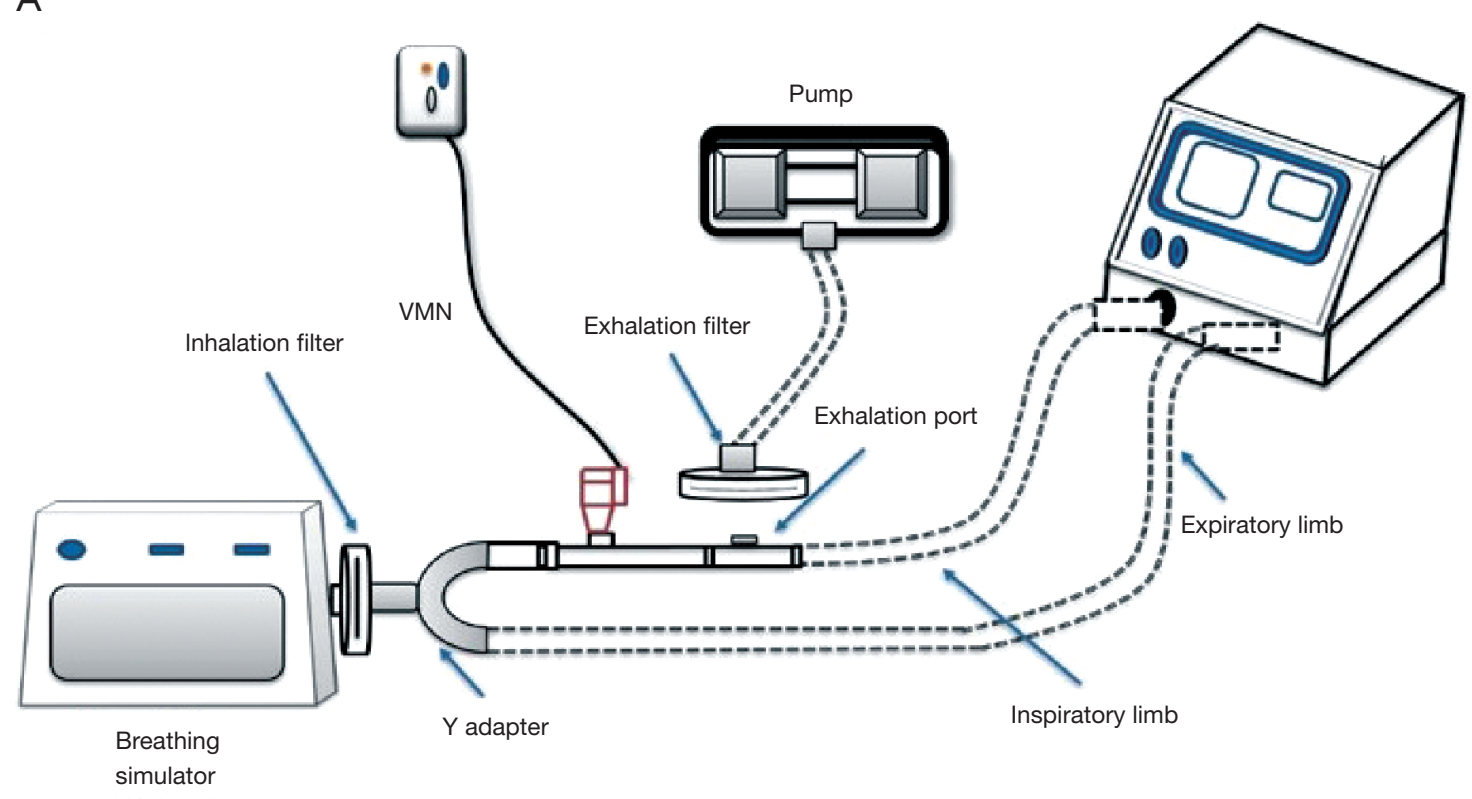

B
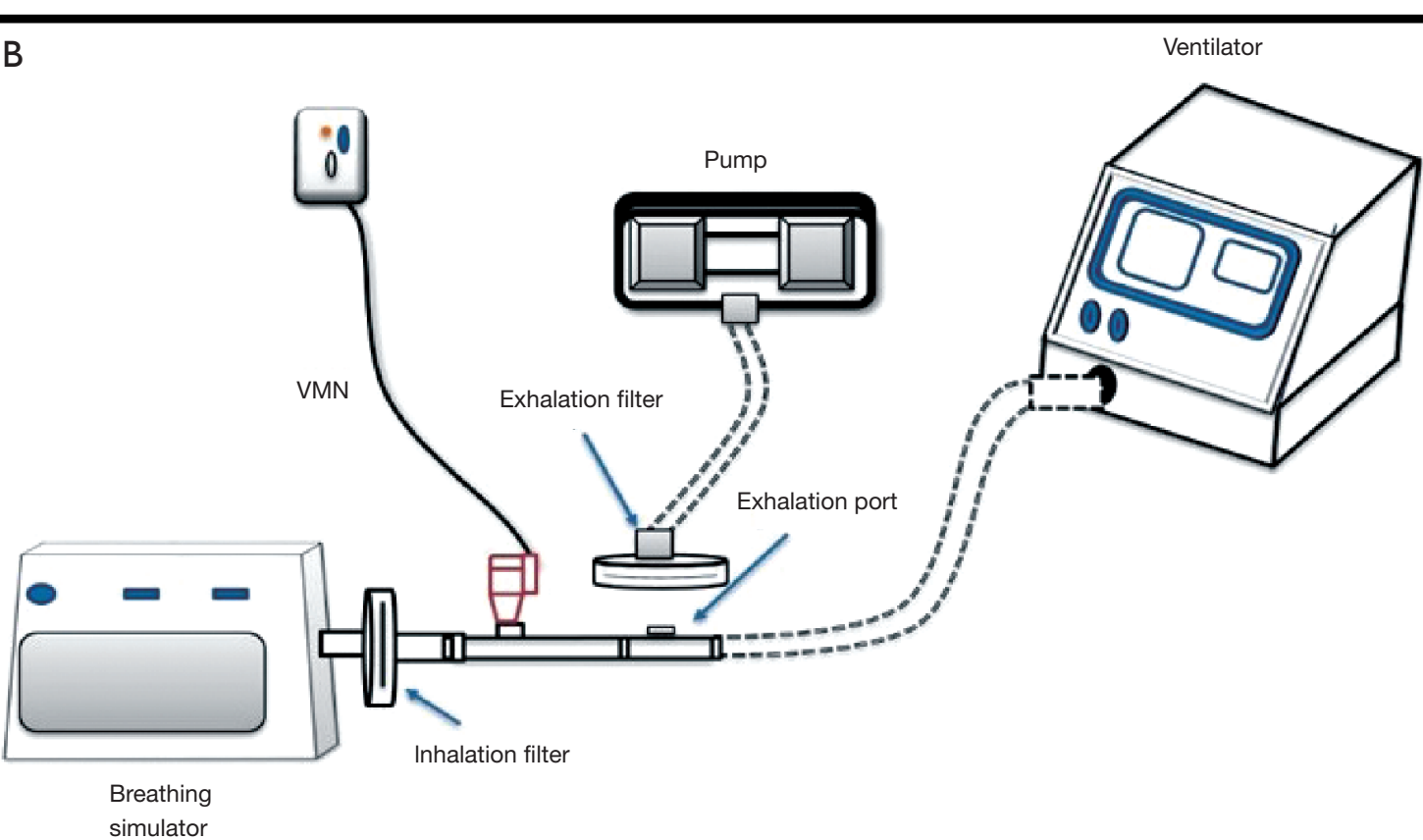

Figure 1 Schematic design of the in vitro model mimicking (A) dual limb and (B) single limb ventilation.

simulator to simulate the patient condition, two T-pieces connected in addition to the cascade impactor. The cascade impactor should be first placed in the refrigerator before use for one hour in case of the ACI while 1.5 hours is needed for the NGI $(16,17)$. The exact arrangement of these parts in a single limb NIV circuit is shown in Figure $2 A$ where
Figure $2 B$ shows that of a dual limb. As noted, the cascade impactor is connected to a vacuum pump to withdraw air at an estimated flow rate of $15 \mathrm{~L} / \mathrm{min}$ which in turn stimulates the patient inspiratory flow rate (quiet breathing pattern) and at the same time allows the entry of an aerosol sample to the cascade impactor device to be analyzed. Particle 
A

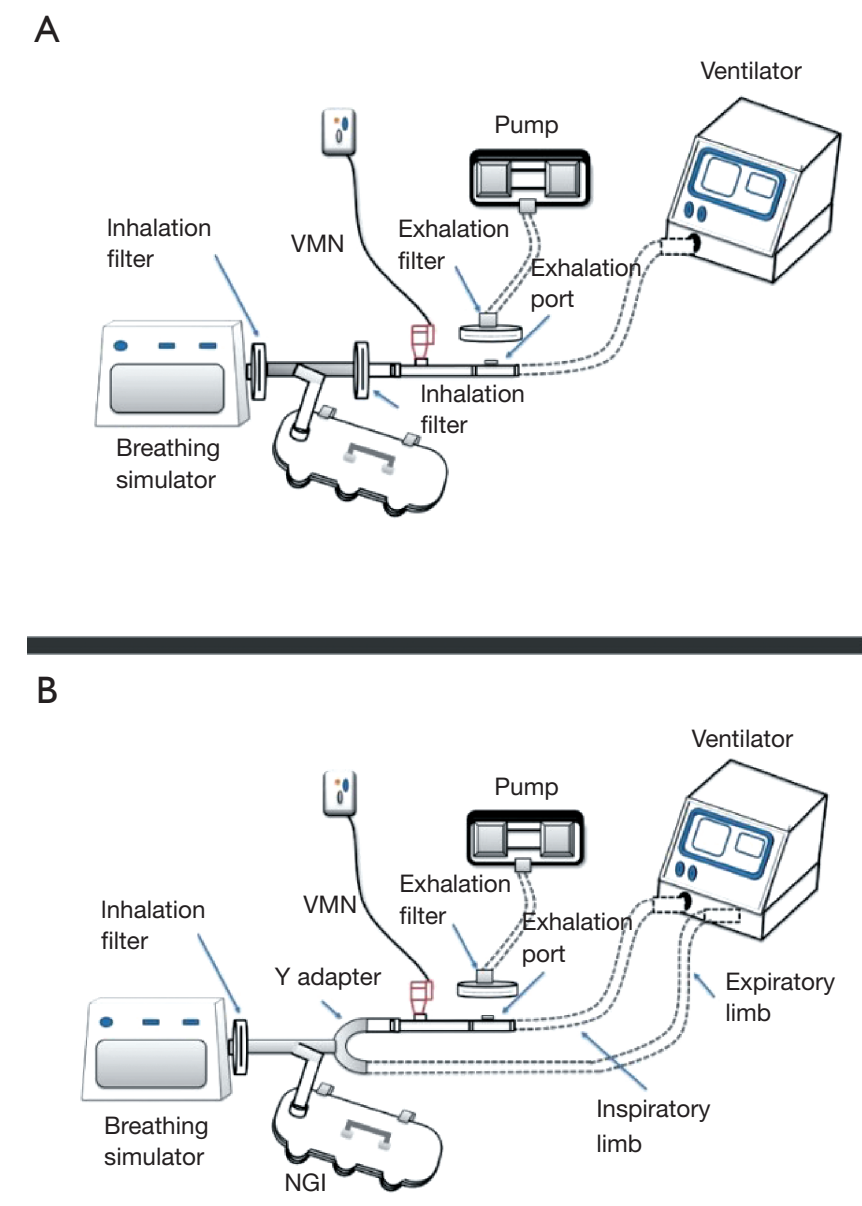

C

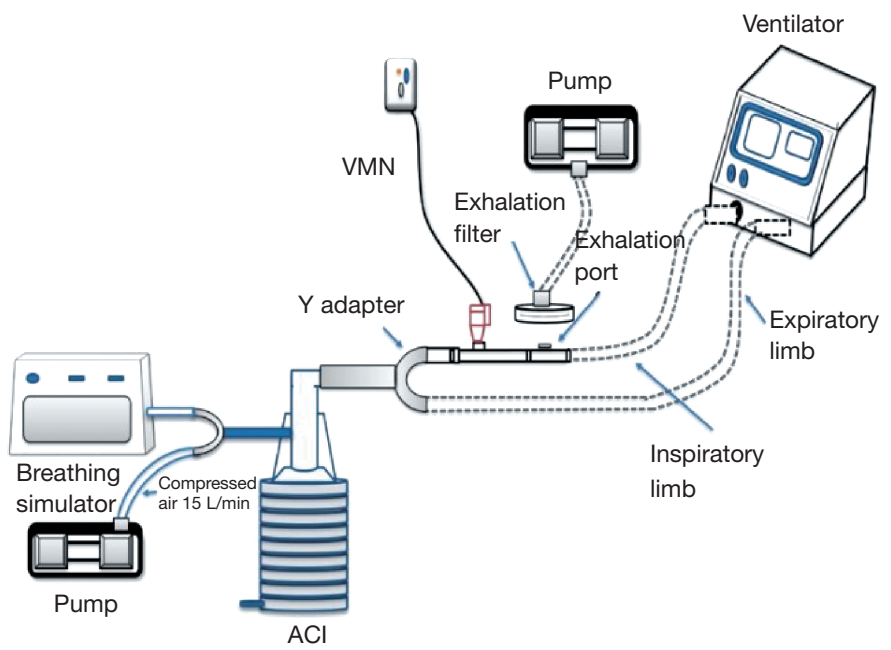

Figure 2 Schematic of breathing simulator and bi-level ventilator circuit for aerodynamic particle size characterization of the respirable dose, (A) single limb, (B) dual limb and (C) for determination of the whole aerosol emitted.

characterization is done only for the entered sample, not all the aerosol emitted which is considered the major drawback of the previously described setup. Hassan et al. determined the aerodynamic characteristics of aerosol droplets released from 4 different nebulizers during NIV using Anderson cascade impactor to measure MMAD, and fine particle fraction (12). The model consisted of a ventilator, breathing simulator, Anderson cascade impactor, and NIV breathing circuit which consisted of a $180 \mathrm{~cm}$ length of corrugated tubing (diameter of $22 \mathrm{~mm}$ ) and a fixed leak expiration port. Although jet nebulizer exhibited a higher fine particle fraction the VMNs delivered the highest fine particle doses (12).

One adjustment can be performed within the NIV circuit to allow the characterization of all delivered aerosol through the use of mixing inlet as shown in Figure $2 C$ where a Y-tube is used to connect a supplementary air delivered at a flow rate of $15 \mathrm{~L} / \mathrm{min}$, breathing simulator and the mixing inlet to the cascade impactor (18). This adjustment was only tested with a dual limb NIV circuit with the possibility of applying it in a single limb. The presence of this mixing inlet allows additional air to be delivered at an equal flow rate to that withdrawn by the vacuum pump connected to the cascade impactor which in turn results in zero flow rate withdrawn from the ventilation circuit. Using this setup, when the inspiratory phase of the breathing simulator was withdrawn from the supplementary air, the inhalation profile is replayed within the ventilation circuit; and because 
of the constant flow $(15 \mathrm{~L} / \mathrm{min})$ of air being withdrawn through the cascade impactor. Hence, the aerosolized drug can be withdrawn from the circuit which is then withdrawn into the cascade impactor. When the expiratory phase started, it added more airflow to the supplementary air. The inhalation profile again is replayed within the ventilation circuit, because of the constant flow of air being withdrawn through the cascade impactor. So, the aerosolized drug is kept within the circuit till the next inspiratory phase resampling what truly happens when delivering aerosol to the patient (18). This model has been used to determine the emitted dose in a study by Elhansy et al. They conducted an in vitro study comparing VMN and Jet nebulizer with a spacer (18). Similar to what was found in the previous study; Jet nebulizer showed the highest fine particle fraction compared with VMNs, while VMNs showed the highest fine particle doses.

To simulate the human lung, the cascade impactor consists of several stages arranged in a descending manner according to the pore diameter of each stage where the stage with the largest pore diameter is placed first near the device mouth inlet followed by that of lower diameter and so on until reaching the last stage (19). The known cutoff diameters for both ACI and NGI stages are displayed at $28.3,60$, and $90 \mathrm{~L} / \mathrm{min}$. However, other flow rates can be used with ACI but adjustment of the stages cutoff diameters is needed at the newer flow rate which can be done by applying this equation: $\mathrm{ECDF}_{2}=\mathrm{ECD}_{28.3}$ $(28.3 / \mathrm{F} 2)^{0.5} \cdot \mathrm{ECDF}_{2}, \mathrm{ECD}_{28.3}$, and $\mathrm{F}_{2}$ symbols stand for the effective cut off diameters at the newly applied flow, the effective cut off diameters at the standard flow (28.3 L/min), the newer flow in $\mathrm{L} / \mathrm{min}$, respectively (20). As particles pass through cascade impactor, particles with sufficient inertia will impact within a certain stage while the rest of particles pass through the stage pores to the following stages until settling of all emitted particles. The amount of drug deposited on each stage can be eluted by washing the stage with a specific solvent carefully chosen to match the solubility of the studied drug and then mass determination is performed by either high-performance liquid chromatography (HPLC) or ultra-violet (UV) spectrophotometer. Then, the mass values of the measured drug in each stage are entered into certain software supplied by the cascade impactor manufacturer to translate them into the aerodynamic characteristics of the administered aerosol; MMAD, fine particle dose (FPD), fine particle fraction (FPF), and geometric standard deviation (GSD). MMAD is a measure of central tendency and refers to the cutoff size where half particles are smaller than that referred particle size and the other half is higher. FPD refers to the fraction of the dose with particle size lower than $5 \mu \mathrm{m}$ where FDF is simply the percentage of FPD. GSD is used to describe the range of distribution of inhaled particles where the highly destructed or distributed aerosol is indicated by a higher GSD number. As mentioned before, particles of $5 \mu \mathrm{m}$ or more will impact on the oropharyngeal region and can participate in the systemic drug effects if orally absorbed besides increasing its adverse effects. However, particles smaller than $2 \mu \mathrm{m}$ in diameter are mainly deposited in the alveolar region by sedimentation so the inhaled aerosol with smaller MMAD $(<2 \mu \mathrm{m})$ is still preferred for many respiratory diseases.

\section{Ex vivo models}

The ex vivo study can be considered as an intermediate phase between in vitro and in vivo studies where it introduces an additional perspective to the measurement of the total emitted dose over the in vitro model which is the presence of patient to exhibit a realistic breathing pattern and takes into consideration the individual differences. While in vitro models using mechanical breath simulator all runs are similar, the ex vivo model has a natural variability among participating subjects which reflect more representative results. A study demonstrating the effect of fill volume and humidification (in vitro model) on the emitted dose from nebulizer reported results that differed from the ex vivo model for the same factors $(13,21)$. Also, such a study helps the researcher to set correlations between in vitro and in vivo studies (22). Ex vivo models use collecting filters between the aerosol device and patient airway, allowing the determination of inhaled dose to be collecting using actual patient breathing patterns (Figure 3A) (21,23).

Saeed et al. conducted an ex vivo study on 2 types of nebulizers during NIV, 48 subjects were recruited in the study and they were divided into 4 groups which received aerosolized medication from nebulizers under different condition (fill volume and humidification), inhalation filters were placed before the subject facemask to collect the total emitted dose (21). The finding of the study showed a great significance of increasing fill volume on aerosol delivery from both nebulizers, however, there was no significant difference regarding humidification (21). 
A

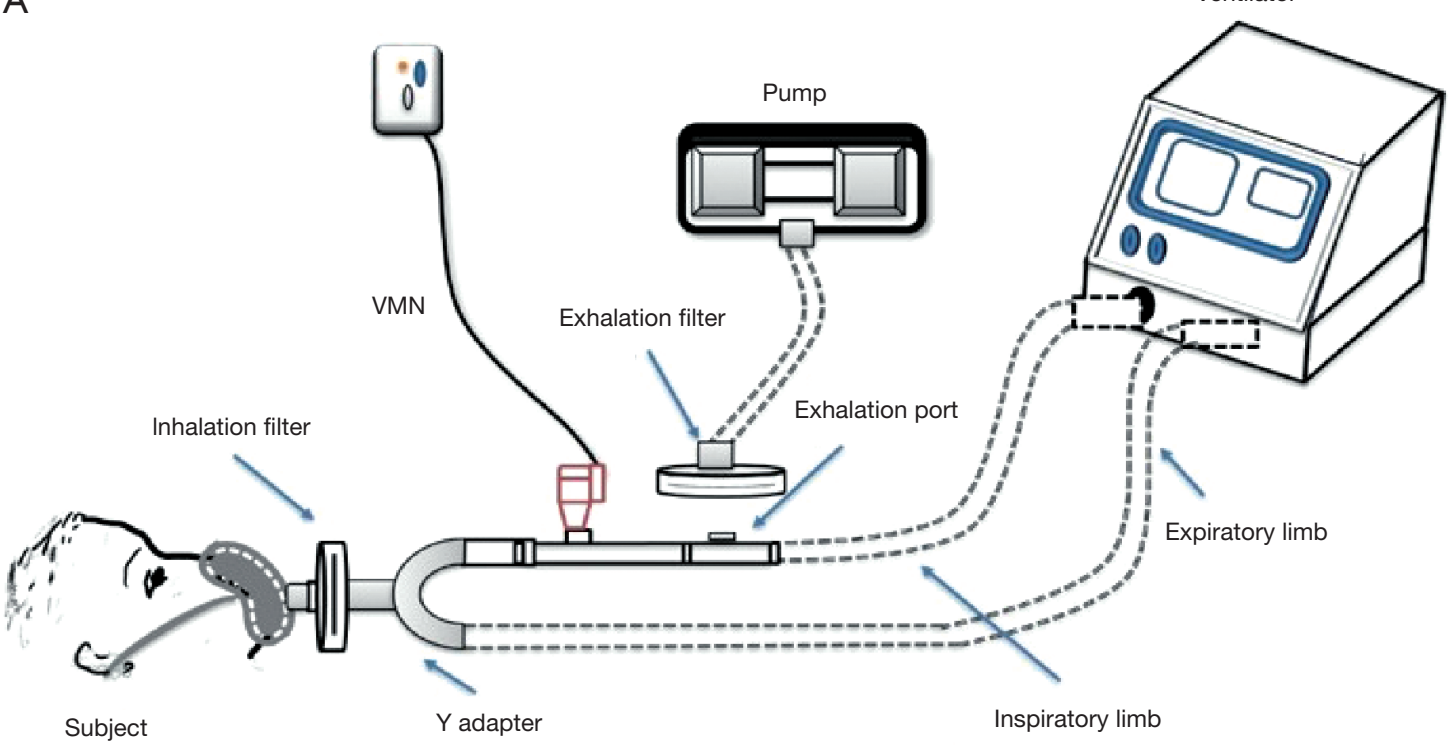

B

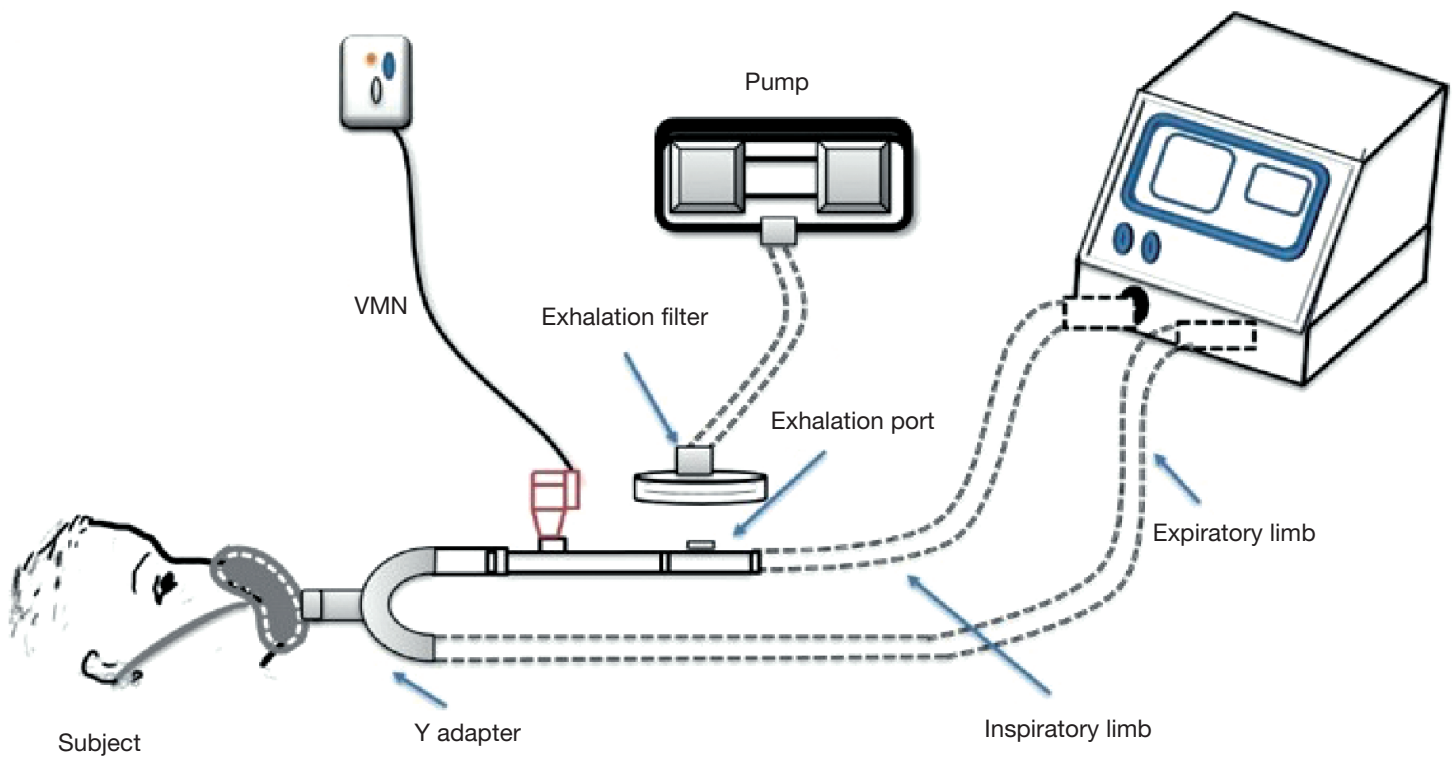

Figure 3 Schematic design of (A) ex vivo and (B) in vivo studies.

\section{In vivo models}

The human subject either a volunteer or a patient is an essential component of these studies where the subject inhales the prescribed drug dose and then biological samples are drawn for further investigations mainly to quantify the inhaled mass and determine the possible drug activity
(Figure 3B). Moreover, certain in vivo models additionally allow the assessment of aerosol distribution within different lung regions. To ensure correct results of the delivered drug dose, temporarily stopping the drug under study to provide a washout period is crucial before the start of the study where the exact stopping period depends on the 
studied drug itself. Of note, a drug alternative should be administered during this period to meet the clinical needs of the patient.

\section{Pharmacokinetics model}

The principle of pharmacokinetic models depends on the analysis of biological samples either plasma (24) or urine samples were taken from the patient after inhaling the prescribed medicine by certain inhaler device during its absorption lag time to quantify the inhaled amount. However, the analysis of blood samples drawn during the drug absorption lag time usually accounts for lower drug concentration because of the larger volume of distribution. Pharmacokinetic models depend on urine analysis, which can be carried out in two different pathways where the first one depends on oral charcoal co-administration with the inhaled drug to adsorb any swallowed fraction of the drug and therefore the drawn urine samples would contain only the delivered fraction to the lung. Unfortunately, the administered charcoal not only blocks the absorption of the drug of interest, but also other drugs are given to the patient which is often seen with ventilated patients. Furthermore, the second urine model pathway mainly relies on the drug absorption lag time where two urine samples are usually taken from the patient (21). In the case of salbutamol bronchodilator, this time is estimated to about $30 \mathrm{~min}$ where the first sample is taken $30 \mathrm{~min}$ postinhalation to indicate the pulmonary salbutamol delivery and the second sample is collected up to 24-hour postinhalation in containers to indicate the systemic delivery. The success of this in vivo determination pathway depends on the drug nebulization time which is preferred not to be more than $8 \mathrm{~min}$ to guarantee that all the lung delivered drug fraction will appear in $30 \mathrm{~min}$ post-inhalation urine sample (25). This estimated nebulization time of $8 \mathrm{~min}$ is considered suitable to nebulize approximately $2 \mathrm{~mL}$ of the drug with conventional JN even with its decreased efficiency compared to other nebulizer types, the higher the fill volume the longer the nebulization time. The volume of the collected samples is recorded, and the drug contained in these samples should be measured by the available analysis methods. HPLC is the most commonly used method with urinary pharmacokinetic models where urine samples are needed to be subjected to prior processing by solid-phase extraction (SPE) before injection into the HPLC device. SPE aims to get rid of unwanted endogenous substances and retains only the drug of interest on specifically chosen cartilage where the existence of these unwanted substances can destruct the HPLC device or eventually mask the desired drug peak. Of note, an additional step is needed when dealing with $24 \mathrm{~h}$ urine samples before SPE is a prehydrolysis step to break down any drug conjugate; ester form or other possible forms into the free drug to be determined (26).

Harb et al. conducted an in vivo study with a pharmacokinetic model to demonstrate the effect of different connections (T-piece and Spacer) on aerosol delivery to 12 patients during NIV (27). A $1-\mathrm{mL}$ solution of salbutamol was nebulized by VMN with 3 settings (I) T-piece, (II) large spacer, and (III) large spacer plus pMDI, 2 urine samples were collected $(0.5$ and $24 \mathrm{~h})$ and analyzed. Findings showed that the T-piece and the spacer were equally efficient for aerosol delivery from VMN in COPD subjects and on single-limb NIV. Also, the addition of 2 puffs of bronchodilators before the nebulization of drugs improved the drug delivery to the lung (27). Hassan et al. using a bi-level ventilator connected to dry singlelimb circuit with the fixed expiratory port were set in spontaneous mode with initial inspiratory to expiratory pressures equal to $20 / 5 \mathrm{cmH}_{2} \mathrm{O}, 15$ breath/minute, and $1: 3$ inspiratory to the expiratory ratio (28). Aerosol generating devices were placed proximal to the facial mask of COPD subjects. $1 \mathrm{~mL}$ of salbutamol solution was nebulized using 2 types of nebulizers (VMN and JN). In vivo amount of drug excreted in urine $(0.5$ and $24 \mathrm{~h})$ was determined after extraction by solid-phase extraction. The finding of the study showed that VMN had higher aerosol delivery compared to the traditional JN (28).

Moustafa et al. studied the effect of humidity on delivered dose in dual limb ventilation circuit and found no significant difference between delivering aerosol with heated humidity and dry conditions (29). This model has been also used to compare the performance of a large spacer versus nebulizer T-Piece in single-limb noninvasive ventilation (27) different vibrating mesh nebulizers in non-invasive ventilation circuit (30), pMDI with different spacers in non-invasive ventilation circuit (31), aerosol delivery during invasive mechanical ventilation (32), aerosol delivery through adult high-flow nasal cannula circuit using low-flow oxygen (33) and in most of the studies they were linked to in vitro, and ex vivo methods for comparison.

Michotte et al. compared continuous and breathsynchronized nebulization of amikacin with a cross over 
design in healthy adult volunteers, they found that the use of inspiratory synchronized vibrating mesh nebulization could enhance pulmonary aerosol delivery compared with the traditional continuous vibrating mesh nebulization (34). This study was an in vivo pharmacokinetic model depend on the collection of the whole urine samples.

\section{Imaging models}

The need for determining regional aerosol deposition within the patient lungs which is mainly affected by its aerodynamic particle size distribution is the driving force for the application of such imaging models. These models can provide reliable data on both the total and the regional pulmonary drug deposition through the use of various imaging techniques such as gamma scintigraphy, singlephoton-emission computed tomography (SPECT), positron emission tomography (PET) and aerosol bolus methods $(35,36)$. Co-administration of the inhaled drug understudy with a radioactive isotope such as Techtanium- $99 \mathrm{~m}$ is the main principle of $2 \mathrm{D}$ gamma scintigraphy where the added isotope is easily detected by the used imaging technique and hence the regional deposition of the inhaled drug can be visualized and assessed $(37,38)$. Many studies used a twodimensional (2D) gamma camera because of its ability to visualize the regional aerosol deposition besides accurate measurement of pulmonary drug deposition through subdivision of lungs into several zones based on the size of the airways $(39,40)$. However, these images do not directly account for the distribution of the inhaled medications throughout the lung (41). Consequently, 3D imaging techniques such as SPECT and PET are greatly appreciated due to their ability to determine the exact mass of aerosol deposited within any pulmonary region and not the relative deposition compared to other regions (42). Their principle as other imaging techniques is based on the use of a radioactive atom administered with the studied drug but this time the gamma camera moves around the patient's lung to collect images from all lung angles (43-45). These images can illustrate the distribution of aerosolized particles on the 3D level of the lungs and also can be used as a tool to differentiate between both peripheral and central deposition of inhaled drugs (43).

França et al. compared radiolabeled aerosol deposition with a jet nebulizer in healthy adults with spontaneous breathing and noninvasive ventilation, reporting no difference in lung deposition, but higher tidal volumes with NIV (46).
Galindo-Filho and colleagues reported a crossover clinical trial involving 10 healthy subjects randomly assigned to received aerosol during NIV with VMN and JN (47). All subjects used naso-oro face masks while receiving positive inspiratory and expiratory pressures of 12 and $5 \mathrm{cmH}_{2} \mathrm{O}$, In a determination of mass balance, $\mathrm{VMN}$ showed a higher drug deposition of inhaled radio-aerosol compared with traditional JNs and higher percentages of radio-aerosol deposited into the lungs. The residual drug volume was higher with JNs compared with VMNs (47). In a similar study in subjects with moderate to severe COPD, the group reported inhaled and lung doses were lower with JN than $\mathrm{VMN}$, also residual drug volume was higher in JN than VMN (48). The peripheral radio-aerosol deposition was significantly higher with VMN compared with JN. It should be noted that while the inhaled dose was similar, the lung deposition was higher with COPD subjects than healthy adults.

\section{Data modeling}

Meaningful data obtained from both in vitro and in vivo models can be gathered and correlated by data modeling technologies for further analysis and optimization. These technologies mainly utilize advanced mathematical and statistical models enabling them to deal with very complex data and multivariate parameters. One of the powerful examples of data mining technologies is artificial neural networks (ANNs), mostly applied in the field of pharmaceutics and pharmaceutical development where their use ensures the ease and speed of results interpretation. Data modeling depends on inputting data into the modeling software which projects and predicts how the experimental results would extend to a greater range of parameters and optimizes the results as shown in Figure 4. ANNs were applied to in vitro data obtained from studies of different drug formulations and medical devices and proved their success in the modeling of these data and predicting the in vivo behavior $(12,31,49-51)$. In noninvasively ventilated patients, this modeling technology was very valuable in comparing aerosol delivery by different inhalers besides the well-known in vitro and in vivo studies $(28,31)$.

Several modeling studies reflected the beneficial application of modeling on merging the data from two or three previously performed studies (in vitro, ex vivo, and in vivo). A study by Saeed et al. demonstrated the effect of nebulizer fill volume on aerosol delivery and deposition in the lung (22). 


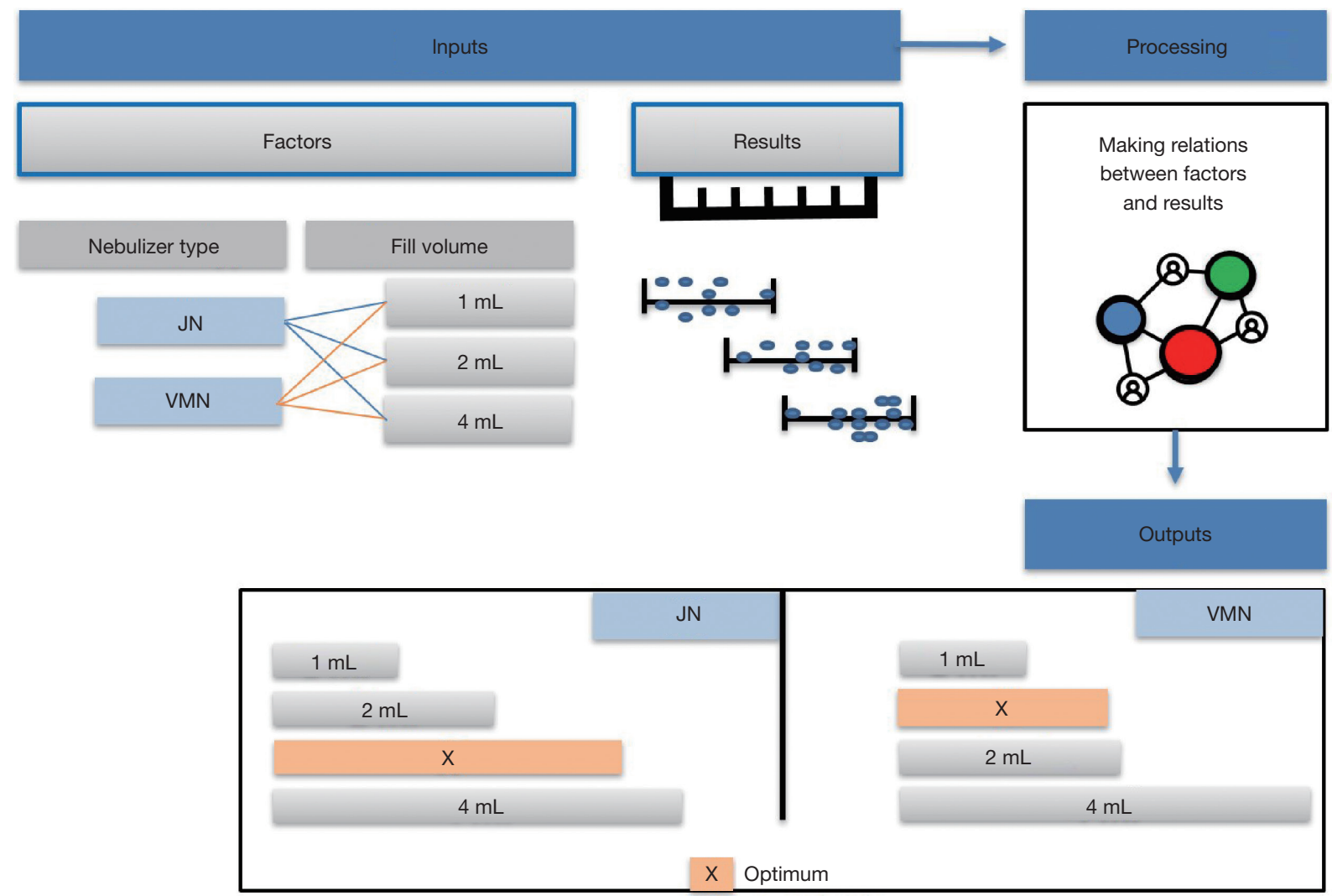

Figure 4 Schematic design of the whole modeling procedure.

The artificial neural networks model showed that VMN increased pulmonary-bioavailability and systemicabsorption compared to the Jet nebulizer. VMN was less affected by fill-volume change compared to Jet nebulizer which should be diluted to increase delivery as shown in Figure 5. The best finding of the modeling was that the model suggested a solution with a nebulizer of the VMN and a fill volume of $2 \mathrm{~mL}$ to achieve the desired lung deposition and systemic absorption which was not significantly found by conventional statistics of the in vitro or the in vivo studies as shown in Figure 6 (22). In Figure $6 \mathrm{~A}$ the contour lines for the in vitro model demonstrated that higher amount of the dose collected in the inhalation filter were obtained with the VMN between 1-2 $\mathrm{mL}$ fill volumes. In Figure $6 B$, the ex vivo collected dose was higher at $2 \mathrm{~mL}$ fill volume with the VMN. Figure $6 C$ and $\mathrm{D}$ shows that the in vivo model demonstrated high collected doses in urine after $30 \mathrm{~min}$ and $24 \mathrm{~h}$ from the VMN at $2 \mathrm{~mL}$ fill volumes. The data of the ex vivo and in vivo models also suggest that increasing the fill volume with the VMN higher than $2 \mathrm{~mL}$ will bring no additional value. Also, the results indicate the presence of differences between the JN and VMN with the favor of the VMN which was less affected by the fill volume changes. In the in vitro, ex vivo, and in vivo studies, they used 3 fill volumes of saline combined with a fixed volume of salbutamol, hence the optimum value should be one of the included 3 volumes, while during the modeling study the required optimum amount of salbutamol was entered to the software to produce a fill volume differ from that was used during the study. The modeling provided better results compared to the traditional statistical models. Hence the main advantage of data modeling is to get more accurate predictions with a small sample size.

Another study by Rabea et al. compared three types of VMNs, which were found equivalent by the conventional statistical method; however, by modeling the in vitro, ex vivo and in vivo data together one of the VMNs was found a bit better than the other two with a suggestion that they are still interchangeable (30). The same was found when modeling the in vitro, ex vivo, and in vivo data of the $\mathrm{pMDI}$ with different spacers within the noninvasive ventilation circuit (31). 
A Inhal. filter (mcg) (Z)

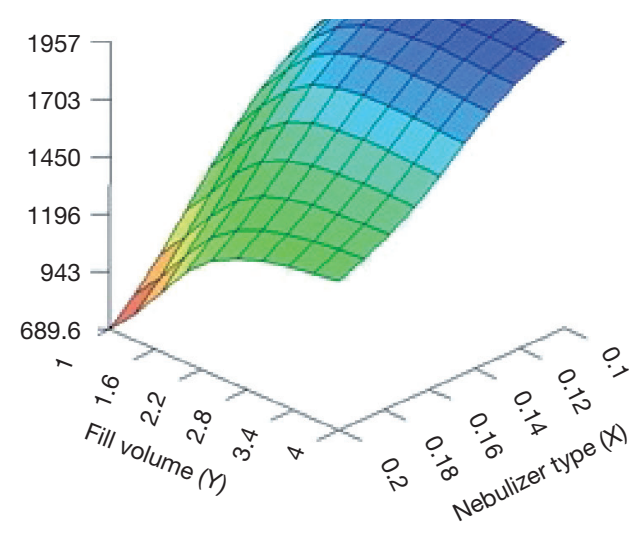

B Ex vivo $(\mathrm{mcg})(\mathrm{Z})$

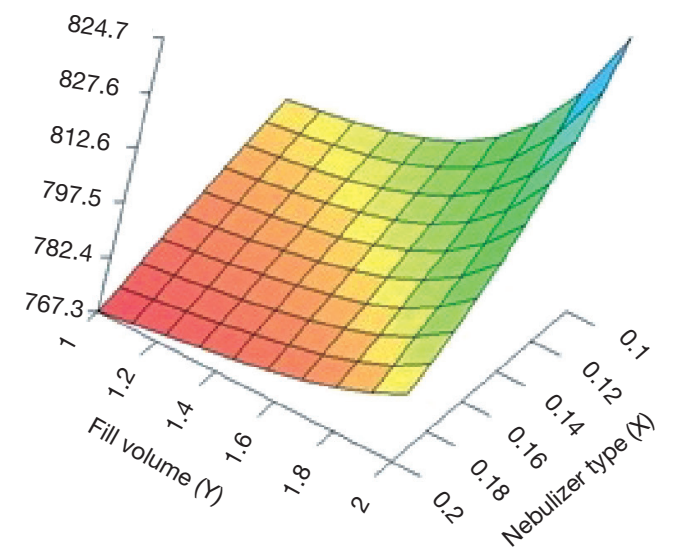

C D/Urine $30 \mathrm{~min}(\mathrm{Z})$

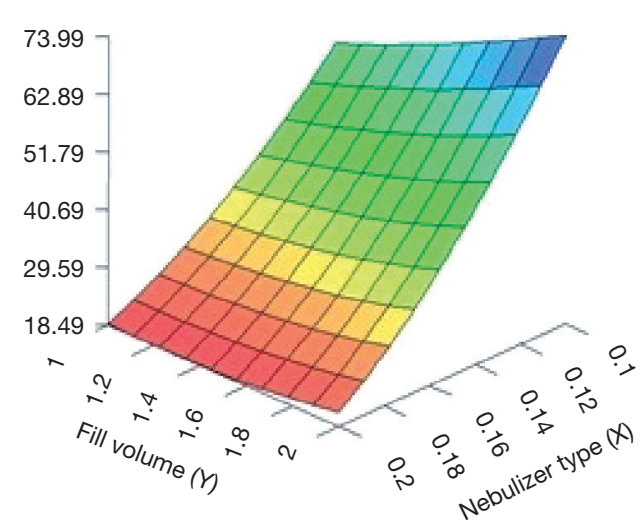

D D/Urine $24 \mathrm{hr}(\mathrm{Z})$

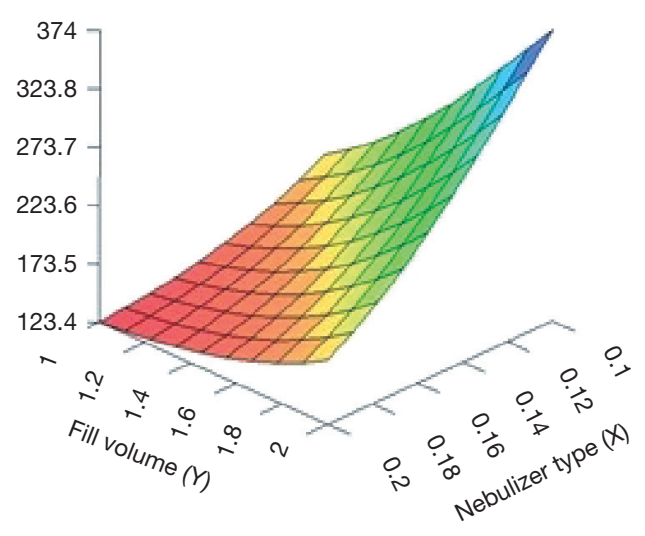

Figure 5 Response surface plots showing effects of nebulizer type and fill volume on (A) amount of drug collected on the inhalation filter, (B) ex vivo results; (C) The amount of drug collected in urine after $30 \mathrm{~min}$ and (D) the amount of drug collected in urine after $24 \mathrm{~h}$ (C \& D) [reproduced from (22)].

So, the modeling was successful in predicting the optimum values of certain factors effectively extending the connection of data points collected from the original studies without performing further experiments (22).

\section{Conclusions}

The methods used to quantify aerosol delivery during NIV are simply in vitro, ex vivo, and in vivo (pharmacokinetic and imaging) methodologies. The in vitro model could be used for the determination of aerosol deposition and aerodynamic characterization, while the in vivo model could be used to reflect the aerosol distribution inside the lung in addition to quantification of the inhaled amount of the drug. Ex vivo studies could provide more realistic results for certain conditions. Modeling of data facilitates the optimization and prediction of more accurate results without further lab studies. 

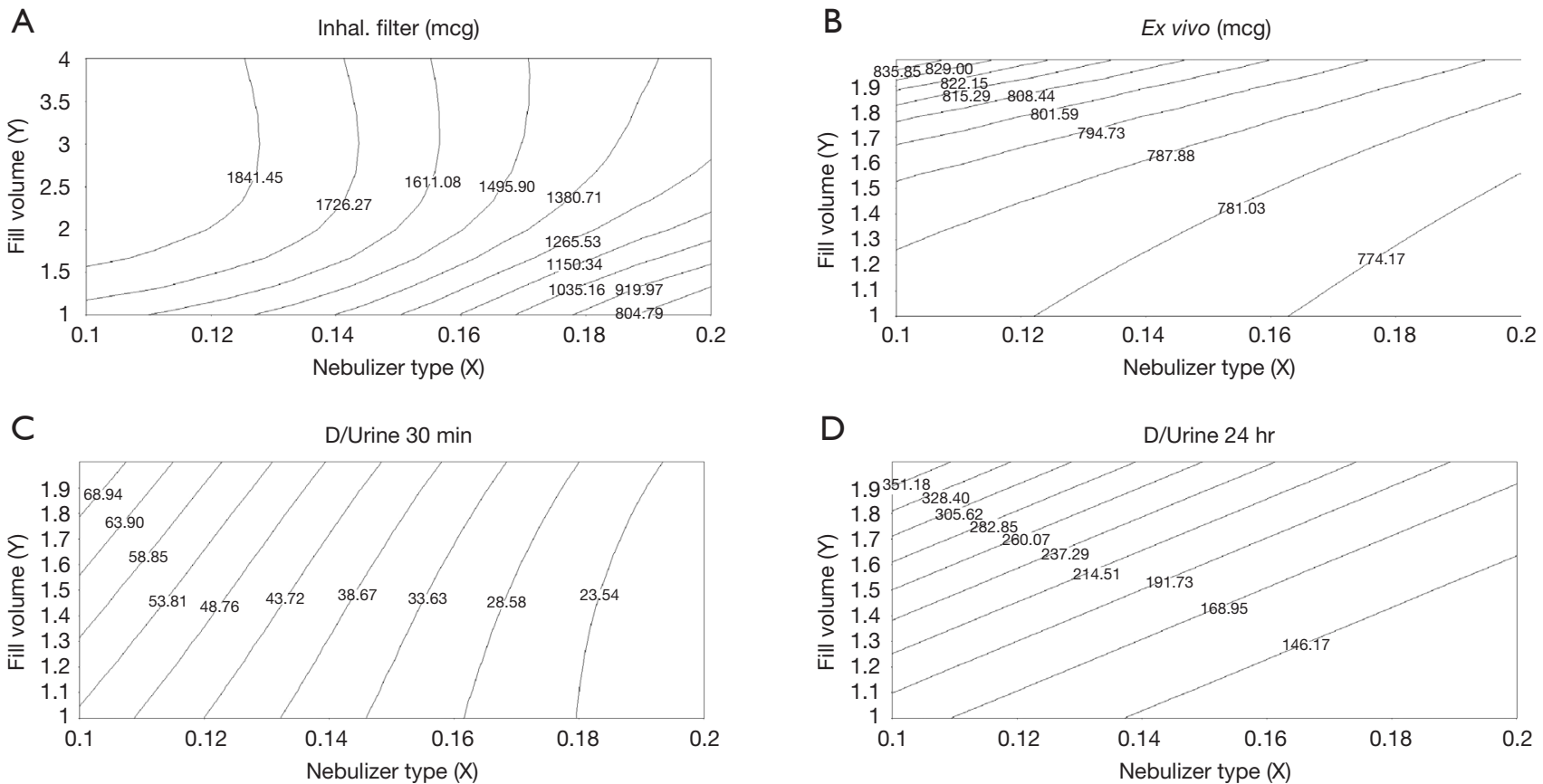

Figure 6 The model generated contour lines for the in vitro collected dose on (A) the inhalation filter, (B) the ex vivo collected dose, the in vivo collected dose in urine (C) after $30 \mathrm{~min}$ and (D) after $24 \mathrm{~h}$ [reproduced from (22)].

\section{Acknowledgments}

Funding: None.

\section{Footnote}

Provenance and Peer Review: This article was commissioned by the Guest Editors (Dr. James B. Fink and Dr. Zhe Luo) for the series "Medical Aerosol in Acute and Critical Care" published in Annals of Translational Medicine. The article has undergone external peer review.

Conflicts of Interest: All authors have completed the ICMJE uniform disclosure form (available at http://dx.doi. org/10.21037/atm-20-1261). The series "Medical Aerosol in Acute and Critical Care" was commissioned by the editorial office without any funding or sponsorship. The authors have no other conflicts of interest to declare.

Ethical Statement: The authors are accountable for all aspects of the work in ensuring that questions related to the accuracy or integrity of any part of the work are appropriately investigated and resolved.
Open Access Statement: This is an Open Access article distributed in accordance with the Creative Commons Attribution-NonCommercial-NoDerivs 4.0 International License (CC BY-NC-ND 4.0), which permits the noncommercial replication and distribution of the article with the strict proviso that no changes or edits are made and the original work is properly cited (including links to both the formal publication through the relevant DOI and the license). See: https://creativecommons.org/licenses/by-nc-nd/4.0/.

\section{References}

1. Darquenne C. Aerosol deposition in health and disease. J Aerosol Med Pulm Drug Deliv 2012;25:140-7.

2. Geller DE. Clinical side effects during aerosol therapy: cutaneous and ocular effects. J Aerosol Med 2007;20:S1008; discussion S109.

3. Brochard L. Mechanical ventilation: invasive versus noninvasive. Eur Respir J Suppl 2003;47:31s-7s.

4. Michotte JB, Jossen E, Roeseler J, et al. In vitro comparison of five nebulizers during noninvasive ventilation: analysis of inhaled and lost doses. J Aerosol Med Pulm Drug Deliv 2014;27:430-40. 
5. Duarte AG. Inhaled bronchodilator administration during mechanical ventilation. Respir Care 2004;49:623-34.

6. Madney YM, Laz NI, Elberry AA, et al. The influence of changing interfaces on aerosol delivery within high flow oxygen setting in adults: An in vitro study. J Drug Deliv Sci Technol 2020;55:101365.

7. Dhand R, Guntur VP. How best to deliver aerosol medications to mechanically ventilated patients. Clin Chest Med 2008;29:277-96.

8. Saeed H, Elberry AA, Eldin AS, et al. Effect of nebulizer designs on aerosol delivery during non-invasive mechanical ventilation: a modeling study of in vitro data. Pulm Ther 2017;3:233-41.

9. Boukhettala N, Porée T, Diot P, et al. In vitro performance of spacers for aerosol delivery during adult mechanical ventilation. J Aerosol Med Pulm Drug Deliv 2015;28:130-6.

10. Bello G, De Pascale G, Antonelli M. Noninvasive ventilation: practical advice. Curr Opin Crit Care 2013;19:1-8.

11. Abdelrahim ME, Plant P, Chrystyn HJJoP, et al. In vitro characterisation of the nebulised dose during non-invasive ventilation. J Pharm Pharmacol 2010;62:966-72.

12. Hassan A, Rabea H, Hussein RRS, et al. In vitro Characterization of the Aerosolized Dose During NonInvasive Automatic Continuous Positive Airway Pressure Ventilation. Pulm Ther 2016;2:115-26.

13. Saeed H, Mohsen M, Fink JB, et al. Fill volume, humidification and heat effects on aerosol delivery and fugitive emissions during noninvasive ventilation. J Drug Deliv Sci Technol 2017;39:372-8.

14. Harb HS, Elberry AA, Rabea H, et al. Is Combihaler usable for aerosol delivery in single limb non-invasive mechanical ventilation? J Drug Deliv Sci Technol 2017;40:28-34.

15. Madney YM, Fathy M, Elberry AA, et al. Nebulizers and spacers for aerosol delivery through adult nasal cannula at low oxygen flow rate: An in vitro study. J Drug Deliv Sci Technol 2017;39:260-5.

16. Abdelrahim ME, Chrystyn H. Aerodynamic characteristics of nebulized terbutaline sulphate using the next generation impactor (NGI) and CEN method. J Aerosol Med Pulm Drug Deliv 2009;22:19-28.

17. Abdelrahim ME. Aerodynamic characteristics of nebulized terbutaline sulphate using the Andersen Cascade Impactor compared to the Next Generation Impactor. Pharm Dev Technol 2011;16:137-45.

18. ElHansy MHE, Boules ME, Farid H, et al. In vitro aerodynamic characteristics of aerosol delivered from different inhalation methods in mechanical ventilation. Pharm Dev Technol 2017;22:844-49.

19. Abdelrahim M, Assi K, Chrystyn H. Dose emission and aerodynamic characterization of the terbutaline sulphate dose emitted from a Turbuhaler at low inhalation flow. Pharm Dev Technol 2013;18:944-9.

20. Van Oort M. In vitro testing of dry powder inhalers. Aerosol Sci Technol 1995;22:364-73.

21. Saeed H, Mohsen M, Eldin AS, et al. Effects of fill volume and humidification on aerosol delivery during single-limb noninvasive ventilation. Respir Care 2018;63:1370-8.

22. Saeed H, Ali AM, Elberry AA, et al. Modeling and optimization of nebulizers' performance in non-invasive ventilation using different fill volumes: comparative study between vibrating mesh and jet nebulizers. Pulm Pharmacol Ther 2018;50:62-71.

23. Lin HL, Fang TP, Cho HS, et al. Aerosol delivery during spontaneous breathing with different types of nebulizersin vitro/ex vivo models evaluation. Pulm Pharmacol Ther 2018;48:225-31.

24. Sangwan S, Agosti JM, Bauer LA, et al. Aerosolized protein delivery in asthma: gamma camera analysis of regional deposition and perfusion. J Aerosol Med 2001;14:185-95.

25. Silkstone VL, Corlett SA, Chrystyn H. Determination of the relative bioavailability of salbutamol to the lungs and systemic circulation following nebulization. Br J Clin Pharmacol 2002;54:115-9.

26. Silkstone VL, Dennis JH, Pieron CA, et al. An investigation of in vitro/in vivo correlations for salbutamol nebulized by eight systems. J Aerosol Med 2002;15:251-9.

27. Harb HS, Elberry AA, Rabea H, et al. Performance of large spacer versus nebulizer T-piece in single-limb noninvasive ventilation. Respir Care 2018;63:1360-9.

28. Hassan A, Salah Eldin R, Abdelrahman MM, et al. In vitro/in vivo comparison of inhaled salbutamol dose delivered by jet nebulizer, vibrating mesh nebulizer and metered dose inhaler with spacer during non-invasive ventilation. Exp Lung Res 2017;43:19-28.

29. Moustafa IO, Ali MR-A, Al Hallag M, et al. Lung deposition and systemic bioavailability of different aerosol devices with and without humidification in mechanically ventilated patients. Heart Lung 2017;46:464-7.

30. Rabea H, Ali AM, Eldin RS, et al. Modelling of in vitro and in vivo performance of aerosol emitted from different vibrating mesh nebulisers in non-invasive ventilation circuit. Eur J Pharm Sci 2017;97:182-91. 
31. Hussein RR, M A Ali A, Salem HF, et al. In vitro/in vivo correlation and modeling of emitted dose and lung deposition of inhaled salbutamol from metered dose inhalers with different types of spacers in noninvasively ventilated patients. Pharm Dev Technol 2017;22:871-80.

32. ElHansy MH, Boules ME, El Essawy AFM, et al. Inhaled salbutamol dose delivered by jet nebulizer, vibrating mesh nebulizer and metered dose inhaler with spacer during invasive mechanical ventilation. Pulm Pharmacol Ther 2017;45:159-63.

33. Madney YM, Fathy M, Elberry AA, et al. Aerosol delivery through an adult high-flow nasal cannula circuit using lowflow oxygen. Respir Care 2019;64:453-61.

34. Michotte JB, Staderini E, Aubriot AS, et al. Pulmonary drug delivery following continuous vibrating mesh nebulization and inspiratory synchronized vibrating mesh nebulization during noninvasive ventilation in healthy volunteers. J Aerosol Med Pulm Drug Deliv 2018;31:33-41.

35. Yeo LY, Friend JR, McIntosh MP, et al. Ultrasonic nebulization platforms for pulmonary drug delivery. Expert Opin Drug Deliv 2010;7:663-79.

36. Tay ZW, Chandrasekharan P, Zhou XY, et al. In vivo tracking and quantification of inhaled aerosol using magnetic particle imaging towards inhaled therapeutic monitoring. Theranostics 2018;8:3676.

37. Ditcham W, Murdzoska J, Zhang G, et al. Lung deposition of 99m Tc-radiolabeled albuterol delivered through a pressurized metered dose inhaler and spacer with facemask or mouthpiece in children with asthma. J Aerosol Med Pulm Drug Deliv 2014;27:S63-75.

38. Devadason SG, Chan H-K, Haeussermann S, et al. Validation of radiolabeling of drug formulations for aerosol deposition assessment of orally inhaled products. J Aerosol Med Pulm Drug Deliv 2012;25:S6-9.

39. Newman SP, Chan H-K. In vitro/in vivo comparisons in pulmonary drug delivery. J Aerosol Med Pulm Drug Deliv 2008;21:77-84.

40. Newman SP, Wilding IR, Hirst PH. Human lung deposition data: the bridge between in vitro and clinical evaluations for inhaled drug products? Int J Pharm

Cite this article as: Saeed H, Harb HS, Madney YM, Abdelrahim MEA. Aerosol delivery via noninvasive ventilation: role of models and bioanalysis. Ann Transl Med 2021;9(7):589. doi: 10.21037/atm-20-1261
2000;208:49-60.

41. Bur M, Henning A, Hein S, et al. Inhalative nanomedicine-opportunities and challenges. Inhal Toxicol 2009;21:137-43.

42. Kim CS. Methods of calculating lung delivery and deposition of aerosol particles. Respir Care 2000;45:695-711.

43. Cryan S-A, Sivadas N, Garcia-Contreras L. In vivo animal models for drug delivery across the lung mucosal barrier. Adv Drug Deliv Rev 2007;59:1133-51.

44. Jaafar-Maalej C, Andrieu V, Elaissari A, et al. Assessment methods of inhaled aerosols: technical aspects and applications. Expert Opin Drug Deliv 2009;6:941-59.

45. Kwok PCL, Wallin M, Dolovich MB, et al. Studies of Radioaerosol Deposition in the Respiratory Tract. Semin Nucl Med 2019;49:62-70.

46. França EE, de Andrade AFD, Cabral G, et al. Nebulization associated with bi-level noninvasive ventilation: analysis of pulmonary radioaerosol deposition. Respir Med 2006;100:721-8.

47. Galindo-Filho VC, Ramos ME, Rattes CS, et al. Radioaerosol pulmonary deposition using mesh and jet nebulizers during noninvasive ventilation in healthy subjects. Respir Care 2015;60:1238-46.

48. Galindo-Filho VC, Alcoforado L, Rattes C, et al. A mesh nebulizer is more effective than jet nebulizer to nebulize bronchodilators during non-invasive ventilation of subjects with COPD: A randomized controlled trial with radiolabeled aerosols. Respir Med 2019;153:60-7.

49. Ali AMA, Abdelrahim MEA. Modeling and optimization of terbutaline emitted from a dry powder inhaler and influence on systemic bioavailability using data mining technology. J Pharm Innov 2014;9:38-47.

50. Jacobson GA, Yee KC, Wood-Baker R, et al. SULT 1A3 single-nucleotide polymorphism and the single dose pharmacokinetics of inhaled salbutamol enantiomers: are some athletes at risk of higher urine levels? Drug Test Anal 2015;7:109-13.

51. Sun Y, Peng Y, Chen Y, et al. Application of artificial neural networks in the design of controlled release drug delivery systems. Adv Drug Deliv Rev 2003;55:1201-15. 